\title{
Time triggered stochastic hybrid system with nonlinear continuous dynamics
}

\author{
Zahra Vahdat $^{1}$ and Abhyudai Singh ${ }^{2}$
}

\begin{abstract}
Time triggered stochastic hybrid systems (TTSHS) constitute a class of piecewise-deterministic Markov processes (PDMP), where continuous-time evolution of the state space is interspersed with discrete stochastic events. Whenever a stochastic event occurs, the state space is reset based on a random map. Prior work on this topic has focused on the continuous-time evolution being modeled as a linear timeinvariant system, and in this contribution, we generalize these results to consider nonlinear continuous dynamics. Our approach relies on approximating the nonlinear dynamics between two successive events as a linear time-varying system and using this approximation to derive analytical solutions for the state space's statistical moments. The TTSHS framework is used to model continuous growth in an individual cell's size and its subsequent division into daughters. It is well known that exponential growth in cell size, together with a sizeindependent division rate, leads to an unbounded variance in cell size. Motivated by recent experimental findings, we consider nonlinear growth in cell size based on a MichaelisMenten function and show that this leads to size homeostasis in the sense that the variance in cell size remains bounded. Moreover, we provide a closed-form expression for the variance in cell size as a function of model parameters and validate it by performing exact Monte Carlo simulations. In summary, our work provides an analytical approach for characterizing moments of a nonlinear stochastic dynamical system that can have broad applicability in studying random phenomena in both engineering and biology.
\end{abstract}

\section{INTRODUCTION}

Nonlinear dynamics is an inherent feature of physical, biological, and engineering systems. These dynamics are sometimes coupled to stochastic events that occur at discrete times and create random jumps in the state space. In this context, work on nonlinear stochastic dynamical systems has focused on their stability, where adaptive models and eventtriggered controllers are designed to address the stability problem by constructing Lyapunov functions [1]-[6].

Building on this theme, here we consider a class of nonlinear stochastic hybrid system (SHS) where the state evolves as per a nonlinear system, and this continuous-time evolution is interspersed with stochastic events that occur at random times $\boldsymbol{t}_{s}, s \in\{1,2, \ldots\}$ (Fig. 1). The time interval between successive events $\boldsymbol{\tau}_{s}=\boldsymbol{t}_{s}-\boldsymbol{t}_{s-1}$ is assumed to be an independent and identically distributed (iid) random variable

\footnotetext{
${ }^{1}$ Z. Vahdat is with the Department of Electrical and Computer Engineering, University of Delaware, Newark, DE USA 19716. e-mail: zahravdeudel.edu

${ }^{2}$ A. Singh is with the Department of Electrical and Computer Engineering, Biomedical Engineering, Mathematical Sciences, Center for Bioinformatics and Computational Biology, University of Delaware, Newark, DE USA 19716. e-mail: absingh@udel.edu
}

that follows an arbitrary continuous positively-valued distribution $g$. The motivation behind this work comes from recent progress on linear time-triggered stochastic hybrid systems (TTSHS), where the state space evolves as per a linear time-invariant system. Not surprisingly, linear continuous dynamics result in exact analytical solutions on moment dynamics, and these systems have found rich applications in diverse fields from network controlled systems [7]-[13], cell biology [14]-[18], neuroscience [19]-[21], nanosensors [22], and modeling of energy grids/smart buildings [23]-[25].

An essential contribution here is to expand results for nonlinear continuous dynamics. Our approach first defines a nominal path, which represents the system's average path between two successive events. Considering small fluctuations around this nominal path due to random jumps in the state space, we linearize the nonlinear dynamics around the nominal path to approximate the continuous dynamics by a linear time-varying system. Working with this approximation, we derive conditions for the existence of the first/second-order statistical moments and their closed-form expression.

The method is illustrated by applying it to the longstanding problem in biology known as cell size homeostasis [26]-[33]. A fundamental aspect of all cellular living systems is the growth in an individual cell's size and subsequent division into daughter cells. How do cells correct size aberrations so that they do not grow abnormally large or small during this process? While an exponential growth in cell size leads to an unbounded variance, our analysis shows that nonlinearities in the cell growth rate play a key role in cell size homeostasis by ensuring a bounded variance in size.

The paper is organized as follows. In Section II, we describe the nonlinear TTSHS model. In Section III, we develop theorems quantifying the first and second-order moments of the state space. The formulation of cell size control as a nonlinear TTSHS is done in Section IV. We discuss the simulations in conjunction with the theoretical results across parameter regimes, followed by a discussion in Section V.

Notation: Throughout this contribution bold symbols represent random variables/stochastic processes. Capital letters indicate a matrix and small letters with a hat denote a vector. Angular brackets $\langle$.$\rangle stand for the expected value operation$ and $\overline{\langle.\rangle}$ stands for the expected value at steady-state. The symbol $\sim$ denotes having a probability distribution of and, the symbol $\otimes$ denotes Kronecker product. Also, vec $\{$.$\} is$ vectorization operator. $I_{n}$ is identity matrix of size $n$. 


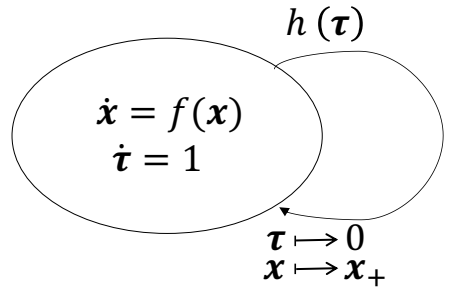

Fig. 1. Schematic of the nonlinear time-triggered stochastic hybrid system (TTSHS). The state of the system evolves as per the nonlinear function (1) and stochastic events occur with rate $h(\boldsymbol{\tau})$ where timer $\boldsymbol{\tau}$ measures the time elapsed since the previous reset. Choosing $h(\tau)$ as per (3) results in time intervals between events following an arbitrary distribution $g$. Moreover, each time an event occurs, the state $\boldsymbol{x}$ undergoes a random jump as per a linear affine random map and the timer $\boldsymbol{\tau}$ resets to zero (4).

\section{NONLINEAR TTSHS MODEl FORMUlation}

Let the state $\boldsymbol{x} \in \mathbb{R}^{n}$ evolve as per the nonlinear system

$$
\dot{\boldsymbol{x}}(t)=f(\boldsymbol{x}(t)),
$$

where function $f: \mathbb{R}^{n} \rightarrow \mathbb{R}^{n}$ is assumed to be a globally Lipschitz continuous on $\mathbb{R}^{n}$ ensuring a unique solution exists $\forall t \geq 0$. This continuous time evolution is interspersed by stochastic resets that are regulated by a timer $\tau$ that measures the time elapsed since the previous reset

$$
\dot{\tau}(t)=1,
$$

and the next reset occurs with rate

$$
h(\tau) \equiv \frac{g(\tau)}{1-\int_{\alpha=0}^{\tau} g(\alpha) d \alpha} .
$$

The resets occur at random times with $h(\tau) d t$ being the probability that a reset occurs in the next infinitesimal time interval $(t, t+d t]$. This timer-based formulation creates memory in reset arrival (for example, a reset becomes more likely to happen as more time elapses since the previous reset).

Let random variable $\tau_{s}$ denote the time between two successive resets, then having a probabilisitic rate as per (3) gauarantes that $\tau_{s}$ is an iid random variable following an arbitrary continuous positively-valued distribution $g$ [10]. With such a formulation, one can capture events like the duration of cell cycle that typically follow a lognormal or gamma distribution [34]-[38]. As expected, the reset's memory-less arrivals are captured by an exponentially-distributed $g$ that corresponds to reset arrival as a per a Poisson process with rate $h=1 /\left\langle\boldsymbol{\tau}_{s}\right\rangle$. Whenever the stochastic reset occurs, the timer is reset to zero and the state changes as per

$$
\boldsymbol{x} \mapsto \boldsymbol{x}_{+}, \boldsymbol{\tau} \mapsto 0,
$$

where random variable $\boldsymbol{x}_{+}$is the state just after the reset (Fig. 1). We assume that $\boldsymbol{x}_{+}$follows a linear affine random map with average (condition on $\boldsymbol{x}$ just before the reset)

$$
\left\langle\boldsymbol{x}_{+} \mid \boldsymbol{x}\right\rangle=J \boldsymbol{x}+\hat{r},
$$

for some matrix $J \in \mathbb{R}^{n \times n}$ and vector $\hat{r} \in \mathbb{R}^{n \times 1}$. Furthermore, the noise in the reset can be represented by the

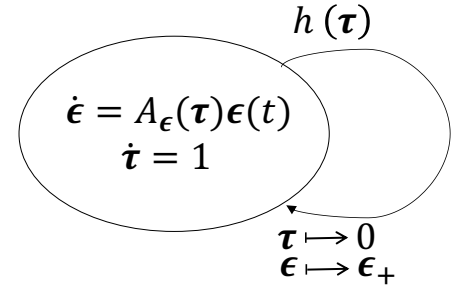

Fig. 2. Approximated linear time-varying time-triggered stochastic hybrid system for the time evolution of the error (10). In this model, the system's state is $\epsilon$ that has linear time-varying dynamics (12) during the time between two stochastic resets. As in the original system in Fig. 1 , the timer measures the time elapsed since the last reset and resets occur probabilistically with rate $h(\tau)$. With each stochastic reset, the timer resets to zero and the state $\epsilon$ jumps to a new value $\epsilon_{+}$as in (13). The statistics of $\epsilon_{+}$are defined by (15) and (16).

covariance matrix of $\boldsymbol{x}_{+}$(condition on $\boldsymbol{x}$ just before the reset)

$$
\begin{aligned}
\left\langle\boldsymbol{x}_{+} \boldsymbol{x}_{+}^{\top} \mid \boldsymbol{x}\right\rangle-\left\langle\boldsymbol{x}_{+} \mid \boldsymbol{x}\right\rangle\left\langle\boldsymbol{x}_{+}^{\top} \mid \boldsymbol{x}\right\rangle= & Q \boldsymbol{x} \boldsymbol{x}^{\top} Q^{\top}+B \boldsymbol{x} \hat{c}^{\top} \\
& +\hat{c} \boldsymbol{x}^{\top} B^{\top}+G,
\end{aligned}
$$

where $Q, B, G \in \mathbb{R}^{n \times n}$ and $\hat{c} \in \mathbb{R}^{n \times 1}$. Intuitively, each reset creates a jump in the state space to a new average value (5), and the noise around this value can be a constant or statedependent. As we will see later, in the context of cell size control, the covariance matrix is state-dependent.

\section{A. Linearizing Nonlinear TTSHS}

To make the nonlinear TTSHS analytically tractable, we linearize the nonlinear continuous dynamics (1) around a nominal path that essentially represents the state space's average path between two successive resets. If $x_{0}$ is the starting state just after a reset, then from (5),

$$
\left\langle J \phi\left(\boldsymbol{\tau}_{s}, x_{0}\right)+\hat{r}\right\rangle
$$

is the average state of the system just after the next reset, where $\phi\left(t, x_{0}\right)$ is the flow of the nonlinear system (1) starting from an initial condition $x_{0}$. Assuming small fluctuations in $\boldsymbol{\tau}_{s}$ around its mean value $\left\langle\boldsymbol{\tau}_{s}\right\rangle$, determining the steady-state value of $x_{0}$ by solving

$$
x_{0} \approx J \phi\left(\left\langle\boldsymbol{\tau}_{s}\right\rangle, x_{0}\right)+\hat{r}
$$

yields the nominal path $\phi\left(\tau, x_{0}\right)$. Recall that timer $\boldsymbol{\tau} \in$ $\left[0, \tau_{s}\right]$ starts from zero measuring the time since the last reset, and itself follows the continuous probability density function (pdf) [10]

$$
\boldsymbol{\tau} \sim p(\tau)=\frac{1}{\left\langle\boldsymbol{\tau}_{s}\right\rangle} \mathrm{e}^{-\int_{0}^{\tau} h(y) d y} .
$$

Next, we define an error

$$
\boldsymbol{\epsilon}(t):=\boldsymbol{x}(t)-\phi\left(\boldsymbol{\tau}, x_{0}\right),
$$

whose continuous dynamic between resets is given by

$$
\dot{\boldsymbol{\epsilon}}(t)=f\left(\phi\left(\boldsymbol{\tau}, x_{0}\right)+\boldsymbol{\epsilon}(t)\right)-f\left(\phi\left(\boldsymbol{\tau}, x_{0}\right)\right) .
$$

Assuming small deviations of $\boldsymbol{x}(t)$ around the nominal path, (11) is approximated by a linear time-varying system

$$
\dot{\boldsymbol{\epsilon}}(t) \approx A_{\epsilon}(\boldsymbol{\tau}) \boldsymbol{\epsilon}(t)
$$


with a Jacobian matrix $A_{\epsilon}(\boldsymbol{\tau}) \in \mathbb{R}^{n \times n}$ obtained by linearizing the vector field $f$ around the nominal path. Having approximated the continuous dynamics of the original nonlinear system with (12), the time evolution of the error can now be modeled as a linear time-varying TTSHS shown in Fig. 2. Whenever a reset occurs, the error and the timer change as per

$$
\epsilon \mapsto \epsilon_{+}, \quad \tau \mapsto 0
$$

with the mean error immediately after reset following

$$
\left\langle\boldsymbol{\epsilon}_{+}\right\rangle=\left\langle\boldsymbol{x}_{+}\right\rangle-x_{0}
$$

Substituting (5) in the above equation and using (10), (14) can be rewritten as (condition on $\boldsymbol{x}$ just before the reset and $\left.\tau_{s}\right)$

$\left\langle\boldsymbol{\epsilon}_{+} \mid \boldsymbol{\epsilon}, \boldsymbol{\tau}_{s}\right\rangle=J \boldsymbol{\epsilon}+\hat{r}_{\epsilon}\left(\boldsymbol{\tau}_{s}\right), \quad \hat{r}_{\epsilon}\left(\boldsymbol{\tau}_{s}\right)=J \phi\left(\boldsymbol{\tau}_{s}, x_{0}\right)+\hat{r}-x_{0}$.

Moreover, the covariance matrix of $\epsilon_{+}$is now given by (condition on $\boldsymbol{x}$ just before the reset and $\tau_{s}$ ), (see Appendix VI-A)

$$
\begin{aligned}
& \left\langle\boldsymbol{\epsilon}_{+} \boldsymbol{\epsilon}_{+}^{\top} \mid \boldsymbol{\epsilon}, \boldsymbol{\tau}_{s}\right\rangle-\left\langle\boldsymbol{\epsilon}_{+} \mid \boldsymbol{\epsilon}, \boldsymbol{\tau}_{s}\right\rangle\left\langle\boldsymbol{\epsilon}_{+}^{\top} \mid \boldsymbol{\epsilon}, \boldsymbol{\tau}_{s}\right\rangle=Q \boldsymbol{\epsilon} \boldsymbol{\epsilon}^{\top} Q^{\top}+\hat{c} \boldsymbol{\epsilon}^{\top} B^{\top} \\
& +B \boldsymbol{\epsilon} \hat{c}^{\top}+Q \boldsymbol{\epsilon} \phi^{\top}\left(\boldsymbol{\tau}_{s}, x_{0}\right) Q^{\top}+Q \phi\left(\boldsymbol{\tau}_{s}, x_{0}\right) \boldsymbol{\epsilon}^{\top} Q^{\top}+G_{\epsilon}\left(\boldsymbol{\tau}_{s}\right)
\end{aligned}
$$

where

$$
\begin{aligned}
G_{\epsilon}\left(\boldsymbol{\tau}_{s}\right)= & Q \phi\left(\boldsymbol{\tau}_{s}, x_{0}\right) \phi^{\top}\left(\boldsymbol{\tau}_{s}, x_{0}\right) Q^{\top}+B \phi\left(\boldsymbol{\tau}_{s}, x_{0}\right) \hat{c}^{\top} \\
& +\hat{c} \phi^{\top}\left(\boldsymbol{\tau}_{s}, x_{0}\right) B^{\top}+G .
\end{aligned}
$$

\section{STATISTICAL MOMENTS OF TTSHS}

In the previous section, we defined a class of nonlinear piecewise-deterministic Markov processes (PDMP) given by (1)-(6) and illustrated in Fig.1. Our purpose is to solve for steady-state first and second-order moments of $\boldsymbol{x}$. We consider the starting point of the nominal path at the steadystate to be $x_{0}$ (8). The system's state starts at $x_{0}$ and evolves with nonlinear dynamics in (1) until a random reset occurs. Given the starting point $x_{0}$, we linearize the nonlinear continuous dynamics and solve the system as linear timevarying TTSHS in (12)-(17) (Fig. 2).

\section{A. Steady-state mean}

Starting from $\epsilon_{0}$, the solution of (12) in the time interval between two resets is

$$
\boldsymbol{\epsilon}(t)=\Phi(\boldsymbol{\tau}, 0) \boldsymbol{\epsilon}_{0}, \quad \Phi(\tau, l)=\Psi(\tau) \Psi^{-1}(l),
$$

where $\Psi(\tau)$ is fundamental matrix and satisfies

$$
\frac{d \Psi(\tau)}{d \tau}=A_{\epsilon}(\tau) \Psi(\tau),|\Psi(.)| \neq 0,
$$

where $|$.$| is determinant operator. The Theorems below$ quantifies the statistical moments for the linear time-varying TTSHS shown in Fig. 2

Theorem 1: The steady-state mean of $\boldsymbol{\epsilon}(t)$ with dynamics expressed in (12)-(15) exists and follows

$$
\overline{\langle\boldsymbol{\epsilon}\rangle}=\langle\Phi(\boldsymbol{\tau}, 0)\rangle\left(I_{n}-J\left\langle\Phi\left(\boldsymbol{\tau}_{s}, 0\right)\right\rangle\right)^{-1}\left\langle\hat{r}_{\epsilon}\left(\boldsymbol{\tau}_{s}\right)\right\rangle,
$$

if and only if $\langle\Phi(\tau, 0)\rangle$ exists and all the eigenvalues of $J\left\langle\Phi\left(\boldsymbol{\tau}_{s}, 0\right)\right\rangle$ are inside the unit circle. $I_{n} \in \mathbb{R}^{n \times n}$ is an identity matrix.

See the proof in the Appendix VI-B. Using the result of Theorem 1 and equation (10), the steady-state mean of $\boldsymbol{x}$ follows

$$
\overline{\langle\boldsymbol{x}\rangle}=\left\langle\phi\left(\boldsymbol{\tau}, x_{0}\right)\right\rangle+\overline{\langle\boldsymbol{\epsilon}\rangle},
$$

where $\left\langle\phi\left(\tau, x_{0}\right)\right\rangle$ will be computed numerically using pdf of $\tau$ in (9).

\section{B. Steady-state second-order moment}

Having the state of the system in (10) composed of two terms, we obtain the second-order moment of the error $\epsilon(t)$. We define a vector $\boldsymbol{\mu}(t)$ that contains moments of the error

$$
\boldsymbol{\mu}(t):=\left[\boldsymbol{\epsilon}^{\top}(t) \operatorname{vec}\left\{\boldsymbol{\epsilon}(t) \boldsymbol{\epsilon}^{\top}(t)\right\}^{\top}\right]^{\top},
$$

where $\operatorname{vec}\{$.$\} is vectorization operator. \boldsymbol{\mu}(t)$ has linear timevarying continuous dynamics interspersed with stochastic resets. Using (12) continuous dynamics of $\boldsymbol{\mu}(t)$ follows

$\dot{\boldsymbol{\mu}}(t) \approx A_{\mu}(\boldsymbol{\tau}) \boldsymbol{\mu}(t), A_{\mu}(\boldsymbol{\tau})=\left[\begin{array}{cc}A_{\epsilon}(\boldsymbol{\tau}) & 0 \\ 0 & 2 A_{\epsilon}(\boldsymbol{\tau})\end{array}\right], \dot{\boldsymbol{\tau}}=1$

where $A_{\epsilon}(\boldsymbol{\tau}) \in \mathbb{R}^{n \times n}$ is in (12) and $A_{\mu}(\boldsymbol{\tau}) \in$ $\mathbb{R}^{\left(n^{2}+n\right) \times\left(n^{2}+n\right)}$. Equation (23) represents homogeneous linear time-varying dynamics. Starting from $\boldsymbol{\mu}_{0}$, the solution of (12) in the time interval between two resets is

$$
\boldsymbol{\mu}(t)=\Phi_{\mu}(\boldsymbol{\tau}, 0) \boldsymbol{\mu}_{0}, \quad \Phi_{\mu}(\tau, l)=\Psi_{\mu}(\tau) \Psi_{\mu}^{-1}(l),
$$

where $\Psi_{\mu}(\tau)$ is fundamental matrix and satisfies

$$
\frac{d \Psi_{\mu}(\tau)}{d \tau}=A_{\mu}(\tau) \Psi_{\mu}(\tau),\left|\Psi_{\mu}(.)\right| \neq 0 .
$$

Now, we rewrite the resets for first and second-order moment of error in matrix form $\boldsymbol{\mu}(t)$ as

$$
\boldsymbol{\mu} \mapsto \boldsymbol{\mu}_{+}, \boldsymbol{\tau} \mapsto 0
$$

where

$$
\left\langle\boldsymbol{\mu}_{+} \mid \boldsymbol{\mu}\right\rangle=J_{\mu}\left(\boldsymbol{\tau}_{s}\right) \boldsymbol{\mu}+\hat{r}_{\mu}\left(\boldsymbol{\tau}_{s}\right)
$$

$J_{\mu}\left(\boldsymbol{\tau}_{s}\right)=\left[\begin{array}{cc}J & 0 \\ B \otimes \hat{c}+\hat{c} \otimes B+ & \\ Q \phi\left(\boldsymbol{\tau}_{s}, x_{0}\right) \otimes Q+ & \\ Q \otimes Q \phi\left(\boldsymbol{\tau}_{s}, x_{0}\right)+ & J \otimes J+Q \otimes Q \\ J \otimes \hat{r}_{\epsilon}\left(\boldsymbol{\tau}_{s}\right)+\hat{r}_{\epsilon}\left(\boldsymbol{\tau}_{s}\right) \otimes J & \end{array}\right]$,

$$
\hat{r}_{\mu}\left(\boldsymbol{\tau}_{s}\right)=\left[\begin{array}{c}
\hat{r}_{\epsilon}\left(\boldsymbol{\tau}_{s}\right) \\
\operatorname{vec}\left(G_{\epsilon}\left(\boldsymbol{\tau}_{s}\right)+\hat{r}_{\epsilon}\left(\boldsymbol{\tau}_{s}\right) \hat{r}_{\epsilon}^{\top}\left(\boldsymbol{\tau}_{s}\right)\right)
\end{array}\right],
$$

$\hat{r}_{\epsilon}\left(\boldsymbol{\tau}_{s}\right)$ and $G_{\epsilon}\left(\boldsymbol{\tau}_{s}\right)$ are in (15) and (17).

We solve for the moment dynamics of error by solving linear time-varying TTSHS system described in (23) and (26)- (27).

Theorem 2: The steady-state solution for $\boldsymbol{\mu}(t)$ with dynamics in (23) and (26)-(27) exists and follows

$\overline{\langle\boldsymbol{\mu}\rangle}=\left\langle\Phi_{\mu}(\boldsymbol{\tau}, 0)\right\rangle\left(I_{n^{2}+n}-\left\langle J_{\mu}\left(\boldsymbol{\tau}_{s}\right) \Phi_{\mu}\left(\boldsymbol{\tau}_{s}, 0\right)\right\rangle\right)^{-1}\left\langle\hat{r}_{\mu}\left(\boldsymbol{\tau}_{s}\right)\right\rangle$, 
if and only if $\left\langle\Phi_{\mu}(\tau, 0)\right\rangle$ exists and all the eigenvalues of $J\langle\Phi(\boldsymbol{\tau}, 0)\rangle$ and $\left((J \otimes J+Q \otimes Q)\left\langle\Phi\left(\boldsymbol{\tau}_{s}, 0\right) \otimes \Phi\left(\boldsymbol{\tau}_{s}, 0\right)\right\rangle\right)$ are inside the unit circle.

Proofs are the same as proofs for Theorem 1. Theorem 2 yields steady-state first and second-order moment of $\epsilon$.

Finally, the steady-state second-order moment of the state follows (see Appendix VI-C for more details)

$$
\begin{aligned}
& \operatorname{vec}\left\{\overline{\left\langle\boldsymbol{x} \boldsymbol{x}^{\top}\right\rangle}\right\}=\operatorname{vec}\left\{\left\langle\phi\left(\boldsymbol{\tau}, x_{0}\right) \phi^{\top}\left(\boldsymbol{\tau}, x_{0}\right)\right\rangle\right\} \\
& +\operatorname{vec}\left\{\overline{\left\langle\boldsymbol{\epsilon} \boldsymbol{\epsilon}^{\top}\right\rangle}\right\}+\operatorname{vvec}\left\{\overline{\left\langle\boldsymbol{\epsilon} \phi^{\top}\left(\boldsymbol{\tau}, x_{0}\right)\right\rangle}\right\},
\end{aligned}
$$

where $\operatorname{vec}\left\{\left\langle\phi\left(\boldsymbol{\tau}, x_{0}\right) \phi^{\top}\left(\boldsymbol{\tau}, x_{0}\right)\right\rangle\right\}$ can be computed numerically using pdf of $\boldsymbol{\tau}$ in (9). Also, we apply Theorem 2 in (30) to compute vec $\left\{\overline{\left\langle\boldsymbol{\epsilon} \boldsymbol{\epsilon}^{\top}\right\rangle}\right\}$, and $\operatorname{vec}\left\{\overline{\left\langle\boldsymbol{\epsilon} \phi^{\top}\left(\boldsymbol{\tau}, x_{0}\right)\right\rangle}\right\}$ is in Appendix VI-C.

\section{Modeling Cell Size Regulation as A NONLINEAR TTSHS}

As an illustrative example of nonlinear TTSHS we consider the problem of cell size growth. Living cells actively proliferate which involves increasing in cell size starting from a newborn cell and then undergoing mitosis (cell division) to create two daughters. Given that the timing of cell division and partitioning of size between daughters has elements of stochasticity, how do individual cells correct size aberrations so that they do not grow abnormally large or small? This problem has been referred to as cell size homeostasis and is an active area of investigation in diverse organisms from microbial to human cells [26], [39]-[51].

Perhaps the simple model of cell growth is one where size of a single cell $\boldsymbol{x}(t)$ increases exponentially over time

$$
\dot{\boldsymbol{x}}(t)=k_{1} \boldsymbol{x}(t), \quad \dot{\boldsymbol{\tau}}=1
$$

and division occurs as per a time with inter-division times $\tau_{s}$. As the cell divides, the timer resets to zero and cell size changes as per

$$
\boldsymbol{x} \mapsto \boldsymbol{x}_{+}, \quad \boldsymbol{x}_{+}=\boldsymbol{b} \boldsymbol{x}, \quad \boldsymbol{\tau} \mapsto 0,
$$

where $\boldsymbol{b}$ is a random variable following the Beta distribution

$$
\boldsymbol{b} \sim \operatorname{Beta}(\alpha, \beta)
$$

where $\alpha$ and $\beta$ are parameters of Beta distribution with mean of $\langle\boldsymbol{b}\rangle$ and variance of $\sigma_{b}^{2}$

$$
\langle\boldsymbol{b}\rangle=\frac{\alpha}{\alpha+\beta}, \quad \sigma_{b}^{2}=\frac{\alpha \beta}{(\alpha+\beta)^{2}(\alpha+\beta+1)} .
$$

In the model, the cell size mean right after reset follows (5). Thereby, $J=1 / 2$ and $\hat{r}=0$

$$
\left\langle\boldsymbol{x}_{+} \mid \boldsymbol{x}\right\rangle=\frac{1}{2} \boldsymbol{x} .
$$

Also, partitioning noise follows (6) with $Q=\sigma_{b}, B=0$, $\hat{c}=0$ and $G=0$

$$
\left\langle\boldsymbol{x}_{+}^{2} \mid \boldsymbol{x}\right\rangle-\left\langle\boldsymbol{x}_{+} \mid \boldsymbol{x}\right\rangle^{2}=\sigma_{b}^{2} \boldsymbol{x}^{2} .
$$

In particular, when the cell size division follows Beta distribution with a mean of $1 / 2$, the parameters should be equal $(\alpha=\beta)$, and the variance is $\sigma_{b}^{2}<1 / 4$. Our prior work has shown that in this case of exponential growth with timerdriven division events

$$
\overline{\langle\boldsymbol{x}\rangle}= \begin{cases}0 & \left\langle\mathrm{e}^{k_{1} \boldsymbol{\tau}_{s}}\right\rangle<2 \\ \infty & \left\langle\mathrm{e}^{k_{1} \boldsymbol{\tau}_{s}}\right\rangle>2\end{cases}
$$

and the only way one could get a non-zero mean cell size is by setting $\left\langle\mathrm{e}^{k_{1} \tau_{s}}\right\rangle=2$ [46]. However, analogous to birthdeath process in the case of $\left\langle\mathrm{e}^{k_{1} \boldsymbol{\tau}_{s}}\right\rangle=2$ even the slightest change causes the variance in cell size to be unbounded [46].

Next, supported by increasing evidence [52]-[56] we consider a nonlinear saturating growth rate

$$
\dot{\boldsymbol{x}}(t)=f(\boldsymbol{x}(t)), \quad f(\boldsymbol{x})=\frac{k_{1} \boldsymbol{x}(t)}{1+k_{2} \boldsymbol{x}(t)}, \quad \dot{\boldsymbol{\tau}}=1
$$

where $k_{1}$ and $k_{2}$ have positive finite values (Fig. 3). This growth rate is exponential when cell size is small and becomes linear when cell size is large.
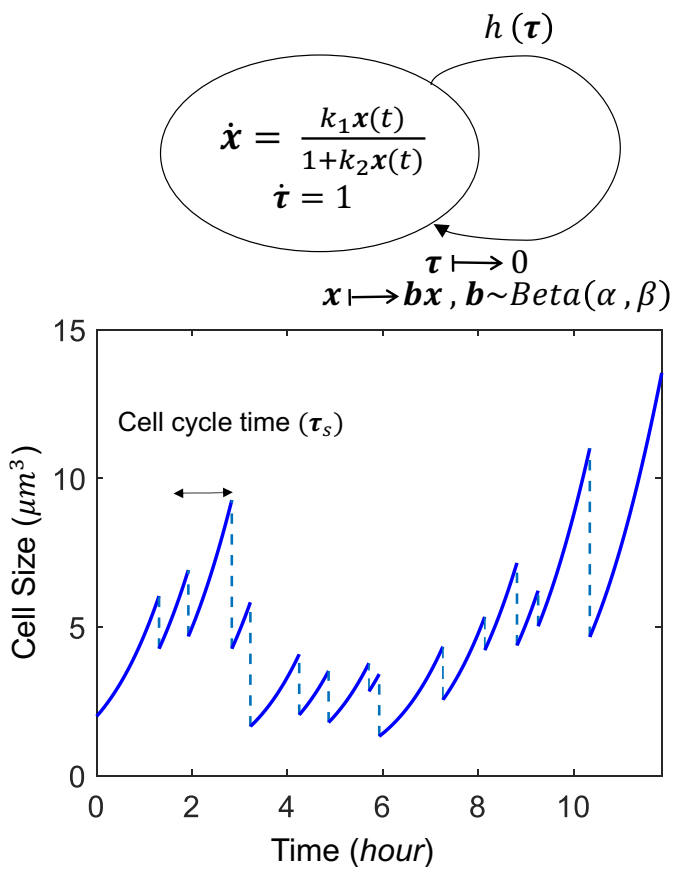

Fig. 3. Top: Time triggered stochastic hybrid model for cell growth and division. Cell grows with a nonlinear function $f(\boldsymbol{x})=\frac{k_{1} \boldsymbol{x}(t)}{1+k_{2} \boldsymbol{x}(t)}$ during each cell cycle and the timer evolves linearly with time. Then cell division occurs with arbitrary hazard rate $h(\boldsymbol{\tau})$. As per the stochastic cell division, the timer resets to zero and cell divides to daughter cells with a random coefficient $\boldsymbol{b}$ following Beta distribution with mean $\frac{1}{2}$ and variance $\sigma_{b}^{2}$. Bottom: Sample realization of the nonlinear TTSHS model. The continuous dynamics follows (1). Cell division hazard rate is $h(\boldsymbol{\tau})=3 \tau^{2}$ and noise in cell division follows Beta distribution with mean $\frac{1}{2}$, and variance $\sigma_{b}^{2}=0.05$ (parameters of Beta distribution are $\alpha=\beta=2$ ). Other parameters are taken as $k_{1}=1$ hour $^{-1}, k_{2}=0.051 / \mu m^{3}$ and $x_{0}=2 \mu \mathrm{m}^{3}$.

By solving (39) when the newborn cell size $x_{0}$ is the starting point of the nominal path, the solution is

$$
\phi\left(\boldsymbol{\tau}, x_{0}\right)=\frac{1}{k_{2}} W\left(k_{2} x_{0} \mathrm{e}^{k_{1} \boldsymbol{\tau}+k_{2} x_{0}}\right),
$$

where $W($.$) is Lambert W$ function or product logarithm function. E.g. if $F(w)=w \mathrm{e}^{w}$, where $w$ is any complex 
number and $\mathrm{e}^{w}$ is the exponential function, then $F^{-1}(w)$ is Lambert $W$ function. By applying (40) in (7) when $\boldsymbol{\tau}=\boldsymbol{\tau}_{s}$ when $J=1 / 2$ and $\hat{r}=0$

$$
x_{0}=\frac{1}{2}\left\langle\phi\left(\boldsymbol{\tau}_{s}, x_{0}\right)\right\rangle=\frac{1}{2 k_{2}}\left\langle W\left(k_{2} x_{0} \mathrm{e}^{k_{1} \boldsymbol{\tau}_{s}+k_{2} x_{0}}\right)\right\rangle .
$$

We simplify the above equation by using the approximation in (8) assuming small noise in $\boldsymbol{\tau}_{s}$, and solving for $x_{0}$

$$
x_{0} \approx \frac{1}{k_{2}}\left(k_{1}\left\langle\boldsymbol{\tau}_{s}\right\rangle-\ln 2\right) \text {. }
$$

So as for the system's starting point of the nominal path $x_{0}$ to be positive, $x_{0}>0, k_{1}>\ln 2 /\left\langle\boldsymbol{\tau}_{s}\right\rangle$. Thereby,

$$
0<\overline{\langle\boldsymbol{x}\rangle}<\infty, \quad k_{2} \neq 0, \quad k_{1}>\ln 2 /\left\langle\boldsymbol{\tau}_{s}\right\rangle,
$$

otherwise, the steady-state mean cell size is zero

$$
\overline{\langle\boldsymbol{x}\rangle}=0, \quad k_{2} \neq 0, \quad k_{1} \leq \ln 2 /\left\langle\boldsymbol{\tau}_{s}\right\rangle .
$$

Thereby by applying (40) in (21) when $k_{2} \neq 0$ and $k_{1}>\ln 2 /\left\langle\boldsymbol{\tau}_{s}\right\rangle$

$$
\overline{\langle\boldsymbol{x}\rangle}=\frac{1}{k_{2}}\left\langle W\left(k_{2} x_{0} \mathrm{e}^{k_{1} \boldsymbol{\tau}+k_{2} x_{0}}\right)\right\rangle+\overline{\langle\boldsymbol{\epsilon}\rangle} .
$$

where now Theorem 1 is used to compute the steady-state mean of the error $\overline{\langle\epsilon\rangle}$. Similarly, using Theorem 2 one can show a finite variance of cell size when $k_{2}>0$, $k_{1}>\ln 2 /\left\langle\boldsymbol{\tau}_{s}\right\rangle$ and $\sigma_{b}^{2}<1 / 4$ (analysis not shown due to space limitations).

As an example $h(\boldsymbol{\tau})$ is chosen to be

$$
h(\boldsymbol{\tau})=3 \tau^{2} .
$$

Therefore, the pdf of $\tau_{s}$ follows Weibull distribution with scale parameter $(\lambda=1)$ and shape parameter $(k=3)$. Also, pdf of timer $\tau$ in (9) is computed using (46).

We compute the steady-state mean of cell size $\overline{\langle\boldsymbol{x}\rangle}$ in (45). We apply numerical integration to compute $\left\langle\phi\left(\tau, x_{0}\right)\right\rangle$, $\left\langle\phi\left(\boldsymbol{\tau}_{s}, x_{0}\right)\right\rangle$ using the density function of $\boldsymbol{\tau}_{s}$ which is $g$ [10] and, the density function of $\tau$ in (9). In Fig. 4(a), we compare the steady-state mean of cell size with simulation results. As shown in the figure, for larger $k_{1}$, the analytical results are close to the mean values obtained by simulation.

Finally, we compute the squared coefficient of variation $C V_{x}^{2}$ for cell size. In Fig. 4(b), we compare the analytic results for noise in cell size with the Monte Carlo simulation results. Keeping the mean value constant, by adjusting $k_{1}$, Fig. 4(b) represents a decrease in noise by increasing $k_{2}$.

\section{CONCLUSION}

In this contribution, we have expanded the tools of approximate moment dynamics to a class of stochastic nonlinear dynamics systems illustrated in Fig. 1. Our approach is still approximate as it relies on small fluctuations in state-space around a nominal path. The key results on existence, and the analytical expression for lower-order statistical moments are summarized in Theorems 1-2, and this approach can be generalized to higher-order moment. The results were applied to the longstanding problem of cell size regulation seeking to characterize intrinsic homeostatic mechanisms that ensure
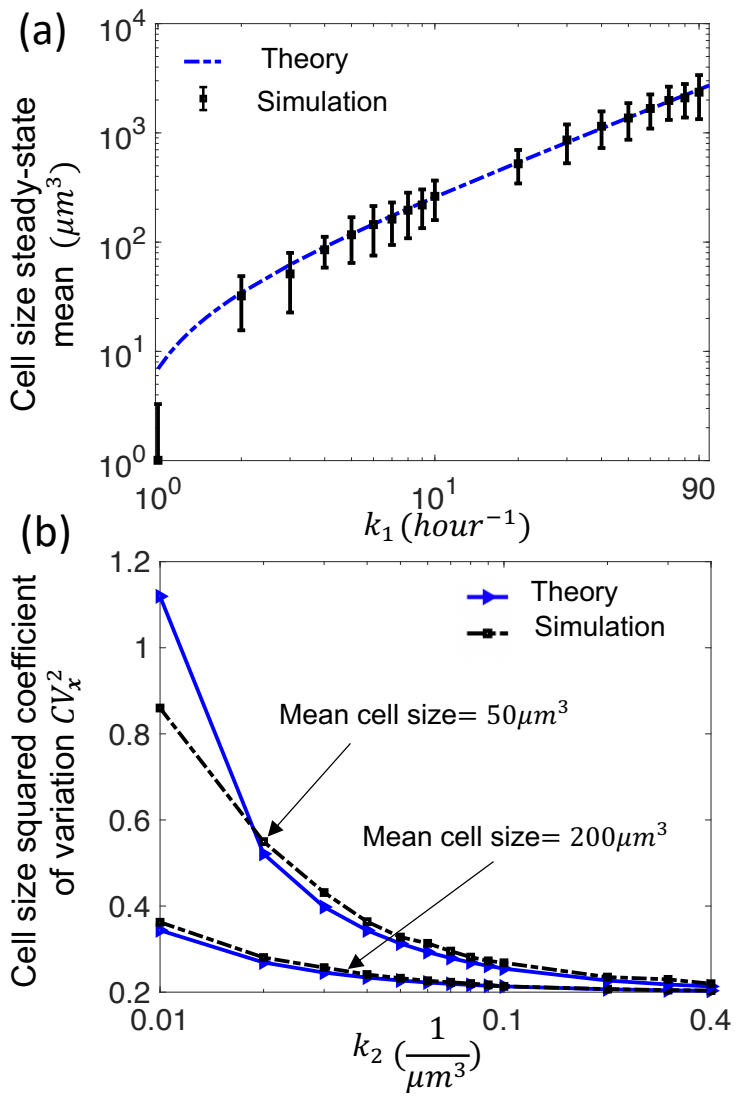

Fig. 4. (a) Comparing cell size mean obtained by theory and Monte Carlo simulation versus growth rate $\boldsymbol{k}_{1}$. Cell size mean increase with increase in $k_{1}$ when $k_{2}=0.051 / \mu \mathrm{m}^{3}$. The results from theory are close to the simulation results when $k_{1}$ is large enough. We performed a Monte Carlo simulation for $10^{5}$ time points and kept the steady-state results. To plot the error bar, we repeated the Monte Carlo with $10^{5}$ time points for 100 times. (b) Comparing cell size noise from the theory and Monte Carlo simulation versus $\boldsymbol{k}_{2} . k_{1}$ is changing so that the steady-state mean value remains constant $\langle\boldsymbol{x}\rangle=200 \mu^{3}$ and $\langle\boldsymbol{x}\rangle=50 \mu \mathrm{m}^{3}$. Also, partitioning noise is $\sigma_{b}^{2}=0.05$ and hazard rate is considered to be $h(\tau)=3 \tau^{2}$. For the analytic results $x_{0}$ is obtained from (42) for each pair of $k_{1}$ and $k_{2}$ Monte Carlo simulation has been done for $10^{5}$ time points.

that sizes of individual cells don't deviate significantly from their target size despite random timing of cell division and random partitioning of size between daughters. Interestingly, our analysis reveals that nonlinearities in cell growth rate, as have been recently reported in [57], are sufficient for cell size homeostasis. More specifically, any non-zero value of $k_{2}$ in (39) with a sufficiently large $k_{1}$ will lead to bounded variance in cell size. These results are illustrated in Fig. 4, where increasing values of $k_{2}$ lead to lower variance in cell size, and analytically predicted moment show an excellent match with similar values obtained from running a large number of Monte Carlo simulations. Future work will further relax the model assumption and consider multi-mode SHS, where the system switches between different continuous dynamics. This is particularly relevant in the context of cell size regulation, where the temporal dynamics of size evolution can be different in different cell-cycle phases. 


\section{APPENDIX}

\section{A. Noise of error just after reset}

We apply $\tau=0$ in (10) to find the state just after reset

$$
\boldsymbol{x}_{+}=x_{0}+\boldsymbol{\epsilon}_{+} .
$$

Using the above equation together with its expectation in (14) and in (6),

$$
\begin{aligned}
& \left\langle\boldsymbol{x}_{+} \boldsymbol{x}_{+}^{\top} \mid \boldsymbol{x}\right\rangle-\left\langle\boldsymbol{x}_{+} \mid \boldsymbol{x}\right\rangle\left\langle\boldsymbol{x}_{+}^{\top} \mid \boldsymbol{x}\right\rangle=\left\langle\left(x_{0}+\boldsymbol{\epsilon}_{+}\right)\left(x_{0}+\boldsymbol{\epsilon}_{+}\right)^{\top} \mid \boldsymbol{\epsilon}\right\rangle- \\
& \left\langle\left(x_{0}+\boldsymbol{\epsilon}_{+}\right) \mid \boldsymbol{\epsilon}\right\rangle\left\langle\left(x_{0}+\boldsymbol{\epsilon}_{+}\right)^{\top} \mid \boldsymbol{\epsilon}\right\rangle=x_{0} x_{0}^{\top}+\left\langle\boldsymbol{\epsilon}_{+} \boldsymbol{\epsilon}_{+}^{\top} \mid \boldsymbol{\epsilon}\right\rangle+x_{0}\left\langle\boldsymbol{\epsilon}_{+}^{\top} \mid \boldsymbol{\epsilon}\right\rangle+ \\
& \left\langle\boldsymbol{\epsilon}_{+} \mid \boldsymbol{\epsilon}\right\rangle x_{0}^{\top}-x_{0} x_{0}^{\top}-x_{0}\left\langle\boldsymbol{\epsilon}_{+}^{\top} \mid \boldsymbol{\epsilon}\right\rangle-\left\langle\boldsymbol{\epsilon}_{+} \mid \boldsymbol{\epsilon}\right\rangle x_{0}^{\top}-\left\langle\boldsymbol{\epsilon}_{+} \mid \boldsymbol{\epsilon}\right\rangle\left\langle\boldsymbol{\epsilon}_{+}^{\top} \mid \boldsymbol{\epsilon}\right\rangle= \\
& \left\langle\boldsymbol{\epsilon}_{+} \boldsymbol{\epsilon}_{+}^{\top} \mid \boldsymbol{\epsilon}\right\rangle-\left\langle\boldsymbol{\epsilon}_{+} \mid \boldsymbol{\epsilon}\right\rangle\left\langle\boldsymbol{\epsilon}_{+}^{\top} \mid \boldsymbol{\epsilon}\right\rangle .
\end{aligned}
$$

Thus, the noise for the defined error just after reset is equal to the noise in the state vector just after reset

$$
\left\langle\boldsymbol{\epsilon}_{+} \boldsymbol{\epsilon}_{+}^{\top} \mid \boldsymbol{\epsilon}\right\rangle-\left\langle\boldsymbol{\epsilon}_{+} \mid \boldsymbol{\epsilon}\right\rangle\left\langle\boldsymbol{\epsilon}_{+}^{\top} \mid \boldsymbol{\epsilon}\right\rangle=Q \boldsymbol{x} \boldsymbol{x}^{\top} Q^{\top}+B \boldsymbol{x} \hat{c}^{\top}+\hat{c} \boldsymbol{x}^{\top} B^{\top}+G \text {. }
$$

Using (10), replacing $\boldsymbol{x}$ as the state just before reset

$$
\boldsymbol{x}=\phi\left(\boldsymbol{\tau}_{s}, x_{0}\right)+\boldsymbol{\epsilon}
$$

in (49) yields (16).

\section{B. Proof of Theorem 1}

Using (18), the mean of the error just before the stochastic reset is

$$
\left\langle\boldsymbol{\epsilon}\left(\boldsymbol{\tau}_{s}\right)\right\rangle=\left\langle\Phi\left(\boldsymbol{\tau}_{\boldsymbol{s}}, 0\right)\right\rangle\left\langle\boldsymbol{\epsilon}_{0}\right\rangle,
$$

and mean of $\epsilon$ just after reset according to (15) is

$$
\left\langle\boldsymbol{\epsilon}_{0}\right\rangle=\left\langle\boldsymbol{\epsilon}_{+}\right\rangle=J\left\langle\boldsymbol{\epsilon}\left(\boldsymbol{\tau}_{s}\right)\right\rangle+J\left\langle\phi\left(\boldsymbol{\tau}_{s}, x_{0}\right)\right\rangle+\hat{r}-x_{0} .
$$

Substituting (51) in (52) and solving for $\left\langle\epsilon_{0}\right\rangle$ yields

$$
\left\langle\boldsymbol{\epsilon}_{0}\right\rangle=\left(I_{n}-J\left\langle\Phi\left(\boldsymbol{\tau}_{s}, 0\right)\right\rangle\right)^{-1} \times\left(J\left\langle\phi\left(\boldsymbol{\tau}_{s}, x_{0}\right)\right\rangle+\hat{r}-x_{0}\right) .
$$

Again by applying (18) at the steady-state we have

$$
\overline{\langle\boldsymbol{\epsilon}\rangle}=\langle\Phi(\boldsymbol{\tau}, 0)\rangle\left\langle\boldsymbol{\epsilon}_{0}\right\rangle,
$$

and finally, substituting (53) in the above equation leads to the result in the Theorem 1 (20).

C. The cell size steady-state second-order moment $\operatorname{vec}\left\{\boldsymbol{x}(t) \boldsymbol{x}^{\top}(t)\right\}$

Following (10), we have

$$
\begin{aligned}
& \boldsymbol{x}(t)=\phi\left(\boldsymbol{\tau}, x_{0}\right)+\boldsymbol{\epsilon}(t), \\
\operatorname{vec}\left\{\boldsymbol{x}(t) \boldsymbol{x}^{\top}(t)\right\}= & \operatorname{vec}\left\{\phi\left(\boldsymbol{\tau}, x_{0}\right) \phi^{\top}\left(\boldsymbol{\tau}, x_{0}\right)\right\}+\operatorname{vec}\left\{\boldsymbol{\epsilon}(t) \boldsymbol{\epsilon}^{\top}(t)\right\} \\
& +2 \operatorname{vec}\left\{\boldsymbol{\epsilon}(t) \phi^{\top}\left(\boldsymbol{\tau}, x_{0}\right)\right\} .
\end{aligned}
$$

Thereby, we first compute the above equation's conditional expectation at the steady-state, and then we take expectation over it, which yields (31). In order to compute the following correlation term in (56)

$$
\boldsymbol{\epsilon}(t) \phi^{\top}\left(\boldsymbol{\tau}, x_{0}\right)=\Phi(\boldsymbol{\tau}, 0) \boldsymbol{\epsilon}_{0} \phi^{\top}\left(\boldsymbol{\tau}, x_{0}\right), \quad \dot{\boldsymbol{\tau}}=1
$$

with the vector form

$$
\operatorname{vec}\left\{\boldsymbol{\epsilon}(t) \phi^{\top}\left(\boldsymbol{\tau}, x_{0}\right)\right\}=\phi\left(\boldsymbol{\tau}, x_{0}\right) \otimes \Phi(\boldsymbol{\tau}, 0) \boldsymbol{\epsilon}_{0},
$$

we apply Theorem 1 with continuous dynamics in (57) and stochastic reset of $\epsilon(t)$ in (13) which yields

$$
\begin{aligned}
& \operatorname{vec}\left\{\overline{\left\langle\boldsymbol{\epsilon} \phi^{\top}\left(\boldsymbol{\tau}, x_{0}\right)\right\rangle}\right\}=\left\langle\phi\left(\boldsymbol{\tau}, x_{0}\right) \otimes \Phi(\boldsymbol{\tau}, 0)\right\rangle \times \\
& \left(I_{n}-J\left\langle\Phi\left(\boldsymbol{\tau}_{s}, 0\right)\right\rangle\right)^{-1}\left(J\left\langle\phi\left(\boldsymbol{\tau}_{s}, x_{0}\right)\right\rangle+\hat{r}-x_{0}\right) .
\end{aligned}
$$

The above correlation exists if and only if $\langle\Phi(\boldsymbol{\tau}, 0)\rangle$ exists and all of the eigenvalues of $J\left\langle\Phi\left(\boldsymbol{\tau}_{s}, 0\right)\right\rangle$ are inside the unit circle.

\section{REFERENCES}

[1] Y. F. Gao, X. M. Sun, C. Wen, and W. Wang, "Event-triggered control for stochastic nonlinear systems," Automatica, vol. 95, pp. 534-538, 2018.

[2] Y. Huang and Y. Liu, "Switching event-triggered control for a class of uncertain nonlinear systems," Automatica, vol. 108, p. 108471, 2019.

[3] M. L. Chiang and L. C. Fu, "Adaptive stabilization of a class of uncertain switched nonlinear systems with backstepping control," Automatica, vol. 50, no. 8, pp. 2128-2135, 2014.

[4] Y. X. Li and G. H. Yang, "Model-based adaptive event-triggered control of strict-feedback nonlinear systems," IEEE transactions on neural networks and learning systems, vol. 29, no. 4, pp. 1033-1045, 2017.

[5] M. M. Polycarpou and P. A. Ioannou, "A robust adaptive nonlinear control design," in 1993 American Control Conference. IEEE, 1993, pp. 1365-1369.

[6] M. Krstic, P. V. Kokotovic, and I. Kanellakopoulos, Nonlinear and adaptive control design. John Wiley \& Sons, Inc., 1995.

[7] A. Singh, Z. Vahdat, and Z. Xu, "Time-triggered stochastic hybrid systems with two timer-dependent resets," OSF Preprints, 2019.

[8] D. Antunes, J. Hespanha, and C. Silvestre, "Volterra integral approach to impulsive renewal systems: Application to networked control," IEEE Transactions on Automatic Control, vol. 57, pp. 607-619, 2012.

[9] J. P. Hespanha, "Modeling and analysis of networked control systems using stochastic hybrid systems," Annual Reviews in Control, vol. 38, pp. 155-170, 2014.

[10] M. Soltani and A. Singh, "Moment analysis of linear time-varying dynamical systems with renewal transitions," SIAM Journal on Control and Optimization, vol. 57, no. 4, pp. 2660-2685, 2019.

[11] J. P. Hespanha, "A model for stochastic hybrid systems with application to communication networks," Nonlinear Analysis: Theory, Methods \& Applications, vol. 62, pp. 1353-1383, 2005.

[12] — "Stochastic hybrid systems: Applications to communication networks," in Hybrid Systems: Computation and Control, R. Alur and G. J. Pappas, Eds. Springer-Verlag, 2004, pp. 387-401.

[13] S. Bohacek, J. P. Hespanha, J. Lee, and K. Obraczka, "A hybrid systems modeling framework for fast and accurate simulation of data communication networks," in Proc. of the ACM Int. Conf. on Measurements and Modeling of Computer Systems (SIGMETRICS), vol. 31, 2003, pp. 58-69.

[14] M. Soltani, C. A. Vargas-Garcia, D. Antunes, and A. Singh, "Intercellular variability in protein levels from stochastic expression and noisy cell cycle processes," PLoS computational biology, vol. 12, no. 8, p. e1004972, 2016.

[15] M. Soltani and A. Singh, "Linear piecewise-deterministic markov processes with families of random discrete events," in 2018 European Control Conference (ECC). IEEE, 2018, pp. 447-452.

[16] D. Antunes and A. Singh, "Quantifying gene expression variability arising from randomness in cell division times," Journal of Mathematical Biology, vol. 71, pp. 437-463, 2015.

[17] A. Singh and J. P. Hespanha, "Stochastic hybrid systems for studying biochemical processes," Philosophical Transactions of the Royal Society A, vol. 368, pp. 4995-5011, 2010.

[18] M. Soltani, C. A. Vargas-Garcia, and A. Singh, "Conditional moment closure schemes for studying stochastic dynamics of genetic circuits," IEEE Transactions on Biomedical Systems and Circuits, vol. 9, pp. 518-526, 2015.

[19] Z. Vahdat, Z. Xu, and A. Singh, "Modeling and characterization of neuronal synapses using stochastic hybrid systems," 2019 IEEE 58th Conference on Decision and Control (CDC), pp. 4729-4734, 2019.

[20] E. Buckwar and M. Riedler, "An exact stochastic hybrid model of excitable membranes including spatio-temporal evolution," Journal of Mathematical Biology, vol. 63, pp. 1051-1093, 2011. 
[21] A. Singh, "Noise mechanisms in synaptic transmission and their impact on spike-timing precision," in 2017 IEEE 56th Annual Conference on Decision and Control (CDC). IEEE, 2017, pp. 5925-5930.

[22] M. Soltani and A. Singh, "Moment-based analysis of stochastic hybrid systems with renewal transitions," Automatica, vol. 84, pp. 62-69, 2017.

[23] M. Strelec, K. Macek, and A. Abate, "Modeling and simulation of a microgrid as a stochastic hybrid system," in 3rd IEEE PES International Conference and Exhibition on Innovative Smart Grid Technologies (ISGT Europe), 2012, pp. 1-9.

[24] A. David, D. Du, K. Larsen, M. Mikučionis, and A. Skou, "An evaluation framework for energy aware buildings using statistical model checking," Science China Information Sciences, vol. 55, pp. 2694-2707, 2012.

[25] J. Hu, "Application of stochastic hybrid systems in power management of streaming data," in Proc. of the 2006 Amer. Control Conference, Minneapolis, $M N$, 2006, pp. 4754-4759.

[26] K. M. Schmoller and J. M. Skotheim, "The biosynthetic basis of cell size control," Trends in cell biology, vol. 25, no. 12, pp. 793-802, 2015.

[27] M. Campos, I. V. Surovtsev, S. Kato, A. Paintdakhi, B. Beltran, S. E. Ebmeier, and C. Jacobs-Wagner, "A constant size extension drives bacterial cell size homeostasis," Cell, vol. 159, pp. 1433-1446, 2014.

[28] C. A. Vargas-Garcia, M. Soltani, and A. Singh, "Conditions for cell size homeostasis: A stochastic hybrid system approach," IEEE Life Sciences Letters, vol. 2, pp. 47-50, 2016.

[29] A. Tzur, R. Kafri, V. S. LeBleu, G. Lahav, and M. W. Kirschner, "Cell Growth and Size Homeostasis in Proliferating Animal Cells," Science, vol. 325, pp. 167-171, 2009.

[30] C. A. Vargas-Garcia, K. R. Ghusinga, and A. Singh, "Cell size control and gene expression homeostasis in single-cells," Current opinion in systems biology, vol. 8, pp. 109-116, 2018.

[31] S. Modi, C. A. Vargas-Garcia, K. R. Ghusinga, and A. Singh, "Analysis of noise mechanisms in cell-size control," Biophysical Journal, vol. 112, pp. 2408-2418, 2017.

[32] J. J. Turner, J. C. Ewald, and J. M. Skotheim, "Cell size control in yeast," Current Biology, vol. 22, pp. R350-R359, 2012.

[33] K. M. Schmoller, J. J. Turner, M. Koivomagi, and J. M. Skotheim, "Dilution of the cell cycle inhibitor Whi5 controls budding-yeast cell size," Nature, vol. 526, pp. 268-272, 2015.

[34] M. Soltani and A. Singh, "Effects of cell-cycle-dependent expression on random fluctuations in protein levels," Royal Society Open Science, vol. 3, p. 160578, 2016.

[35] H. X. Chao, R. I. Fakhreddin, H. K. Shimerov, K. M. Kedziora, R. J. Kumar, J. Perez, J. C. Limas, G. D. Grant, J. G. Cook, G. P. Gupta et al., "Evidence that the human cell cycle is a series of uncoupled, memoryless phases," Molecular systems biology, vol. 15, no. 3, p. e8604, 2019.

[36] P. Costa del Amo, B. Debebe, M. Razavi-Mohseni, S. Nakaoka, A. Worth, D. Wallace, P. Beverley, D. Macallan, and B. Asquith, "The rules of human t cell fate in vivo," Frontiers in immunology, vol. 11, p. 573, 2020.

[37] M. Mura, C. Feillet, R. Bertolusso, F. Delaunay, and M. Kimmel, "Mathematical modelling reveals unexpected inheritance and variability patterns of cell cycle parameters in mammalian cells," PLoS computational biology, vol. 15, no. 6, p. e1007054, 2019.

[38] E. B. Stukalin, I. Aifuwa, J. S. Kim, D. Wirtz, and S. Sun, "Agedependent stochastic models for understanding population fluctuations in continuously cultured cells," Journal of the Royal Society Interface, vol. 10, p. 20130325, 2013.

[39] J. J. Turner, J. C. Ewald, and J. M. Skotheim, "Cell Size Control in Yeast," Current Biology, vol. 22, pp. R350-R359, 2012.

[40] M. B. Ginzberg, R. Kafri, and M. Kirschner, "On being the right (cell) size," Science, vol. 348, p. 1245075, 2015.

[41] M. Osella, S. J. Tans, and M. C. Lagomarsino, "Step by step, cell by cell: quantification of the bacterial cell cycle," Trends in microbiology, vol. 25, no. 4, pp. 250-256, 2017.

[42] S. Son, A. Tzur, Y. Weng, P. Jorgensen, J. Kim, M. W. Kirschner, and S. R. Manalis, "Direct observation of mammalian cell growth and size regulation," Nature methods, vol. 9, no. 9, pp. 910-912, 2012.

[43] A. Lambert, A. Vanhecke, A. Archetti, S. Holden, F. Schaber, Z. Pincus, M. T. Laub, E. Goley, and S. Manley, "Constriction rate modulation can drive cell size control and homeostasis in c. crescentus," Iscience, vol. 4, pp. 180-189, 2018.
[44] Y. Tanouchi, A. Pai, H. Park, S. Huang, R. Stamatov, N. E. Buchler, and L. You, "A noisy linear map underlies oscillations in cell size and gene expression in bacteria," Nature, vol. 523, pp. 357-360, 2015.

[45] I. Soifer, L. Robert, and A. Amir, "Single-cell analysis of growth in budding yeast and bacteria reveals a common size regulation strategy," Current Biology, vol. 26, pp. 356-361, 2016.

[46] C. A. Vargas-García, M. Soltani, and A. Singh, "Conditions for cell size homeostasis: A stochastic hybrid systems approach," IEEE Life Sciences Letters, vol. 2, pp. 47-50, 2016.

[47] J. Hu, J. Lygeros, and S. Sastry, "Modeling subtilin production in bacillus subtilis using stochastic hybrid systems," in Hybrid Systems: Computation and Control. Springer, 2004, pp. 417-431.

[48] E. Cinquemani, R. Porreca, G. Ferrari-Trecate, and J. Lygeros, "Subtilin production by bacillus subtilis: Stochastic hybrid models and parameter identification," IEEE Transactions on Automatic Control, vol. 53, pp. 38-50, 2008.

[49] D. Riley, X. Koutsoukos, and K. Riley, "Modelling and analysis of the sugar cataract development process using stochastic hybrid systems," IET Systems Biology, vol. 3, pp. 137-154, 2009.

[50] C. A. Vargas-García and A. Singh, "Elucidating cell size control mechanisms with stochastic hybrid systems elucidating cell size control mechanisms with stochastic hybrid systems," IEEE Conference on Decision and Control (CDC), 2018.

[51] A. C. Chien, N. S. Hill, and P. A. Levin, "Cell size control in bacteria," Current Biology, vol. 22, pp. R340-R349, 2012.

[52] G. E. Neurohr, R. L. Terry, J. Lengefeld, M. Bonney, G. P. Brittingham, F. Moretto, T. P. Miettinen, L. P. Vaites, L. M. Soares, J. A. Paulo et al., "Excessive cell growth causes cytoplasm dilution and contributes to senescence," Cell, vol. 176, no. 5, pp. 1083-1097, 2019.

[53] M. B. Ginzberg, N. Chang, H. D’Souza, N. Patel, R. Kafri, and M. W. Kirschner, "Cell size sensing in animal cells coordinates anabolic growth rates and cell cycle progression to maintain cell size uniformity," Elife, vol. 7, p. e26957, 2018.

[54] R. Kafri, J. Levy, M. B. Ginzberg, S. Oh, G. Lahav, and M. W. Kirschner, "Dynamics extracted from fixed cells reveal feedback linking cell growth to cell cycle," Nature, vol. 494, pp. 480-483, 2013.

[55] A. Tzur, R. Kafri, V. S. LeBleu, G. Lahav, and M. W. Kirschner, "Cell growth and size homeostasis in proliferating animal cells," Science, vol. 325 , no. 5937, pp. 167-171, 2009.

[56] F. R. Cross and J. G. Umen, "The Chlamydomonas cell cycle," The Plant Journal: For Cell and Molecular Biology, vol. 82, pp. 370-392, 2015.

[57] C. A. Vargas-Garcia, M. Björklund, and A. Singh, "Modeling homeostasis mechanisms that set the target cell size," Scientific Reports, vol. 10 , no. 1 , pp. $1-8,2020$. 